\title{
Computing the safe working zone of a 3-RRS parallel manipulator
}

\author{
Dhruvesh Patel ${ }^{1}$, Rohit Kalla ${ }^{2}$, Halil Tetik ${ }^{3}$, Gökhan Kiper ${ }^{4}$, Sandipan \\ Bandyopadhyay ${ }^{5}$ \\ ${ }^{1}$ Indian Institute of Technology Madras, e-mail: dhruvdplayer@gmail.com \\ 2 Indian Institute of Technology Madras, e-mail: rohitkalla9@gmail.com \\ ${ }^{3}$ Izzmir Institute of Technology, e-mail: haliltetik1989@gmail.com \\ 4 Izmir Institute of Technology, e-mail: gokhankiper@iyte.edu.tr \\ ${ }^{5}$ Indian Institute of Technology Madras, e-mail: sandipan@iitm.ac.in
}

\begin{abstract}
Determination of the safe working zone (SWZ) of a parallel manipulator is a one-time computational task with several permanent benefits. As this subspace of the workspace of the manipulator is free of both the loss- and gain-type singularities, link interference, as well as physical joint limits, the manipulator can move freely in this space. Moreover, if the natural choice of a convex-shaped SWZ is adhered to, then point-to-point path planning inside the SWZ always has a trivial solution, namely, a segment joining the two points, which is guaranteed to be inside the workspace. In this paper, the SWZ of the 3-RRS existing in the İzmir Institute of Technology has been computed. Starting with the geometry of the manipulator, the loop-closure constraint equations have been derived. The singularity conditions are obtained based on the singularity of certain Jacobian matrices associated with the constraint functions. The interference between the links are detected by first encapsulating the links in rectangular parallelepipeds, which are then discretized into triangles, and subjected to collision tests between the relevant pairs of triangles. Using these theoretical developments, the SWZ is computed. The numerical results are depicted graphically.
\end{abstract}

Key words: Parallel manipulator, 3-RRS, Singularity, Safe working zone

\section{Introduction}

Parallel manipulators pose a number of difficulties in terms of applications, as they have complicated workspace shapes, and have singularities inside the workspace as well. Furthermore, they have relatively large number of links, leading to greater possibilities of interference between the links. Any pathplanning procedure needs to take into cognizance all these issues.

One possible solution to the above problems is to compute a subspace of the workspace of the manipulator, in which it is free of all of the above issues. This approach was presented in [4], wherein such a space was termed as the "safe working zone" (SWZ) of a parallel manipulator. The advantage of this approach is that it involves a one-time computation of the SWZ, and thereafter the task of path-panning becomes significantly easier. 
In this work, a 3-RRS manipulator is studied for its SWZ. The manipulator has three degrees of freedom, namely, roll, pitch, and heave. It has been studied in $[5,2]$ for its kinematics etc. In this work, the singularity conditions are derived afresh from the loop-closure equations of the manipulator, and used in the formulation of the SWZ. In order to detect self-collision, the links are encapsulated in cuboid bounding boxes, whose faces are decomposed into two triangles each. Link interference is therefore detected by checking the intersections between triangles, following [1], which is adopted in this work. Using these formulations, the SWZ is computed in a C++ code, which determines the SWZ in less than an hour, with a linear resolution of $0.5 \mathrm{~mm}$, and an angular resolution of $0.125^{\circ}$, on a standard PC. The dimensions etc. are adopted from the existing 3-ㅌRS manipulator in the Mechatronics Laboratory of İzmir Institute of Technology, and the knowledge of the SWZ is going to facilitate further study of the manipulator, as well as its practical applications.

The rest of the paper is organised as follows: the kinematic formulation of the manipulator is discussed in Section 2. Section 3 describes the detailed formulation for finding SWZ for 3-ㅌRS manipulator, based on which the computed SWZ is described in Section 4. The paper is concluded in Section 5.

\section{Kinematic formulation}

The geometry of the 3-RRS manipulator is discussed in this section, followed by the derivation of the loop-closure equations.

\subsection{Geometry}

The 3-RRS manipulator consists of a fixed base and a moving platform, both in the shape of equilateral triangles with circumradii $b$ and $a$, respectively. The triangular platforms are connected by three identical RRS legs (see Fig. 1b). In each leg, the first rotary joint situated at $\boldsymbol{b}_{i}$ is active (i.e., actuated), and the next rotary joint at $\boldsymbol{a}_{i}$ is passive (i.e., not actuated). The legs meet the moving top platform through spherical joints, at the points $\boldsymbol{p}_{i}$. The active link has a length $l$, while the passive one has $r$ as its length. The active joint variables are grouped together as $\boldsymbol{\theta}=\left\{\theta_{1}, \theta_{2}, \theta_{3}\right\}^{\top}$, and the passive joints as $\phi=\left\{\phi_{1}, \phi_{2}, \phi_{3}\right\}^{\top}$. The end-effector pose is parametrized by the variables $\boldsymbol{x}=\{\alpha, \beta, z\}^{\top}$, where $\alpha, \beta$ are the roll and pitch angles, respectively, while $z$ denotes the heave.

The fixed-frame is attached at the base, as shown in Fig 1, and all the vectors are expressed in this frame. The vertices of the triangular base are given by: 


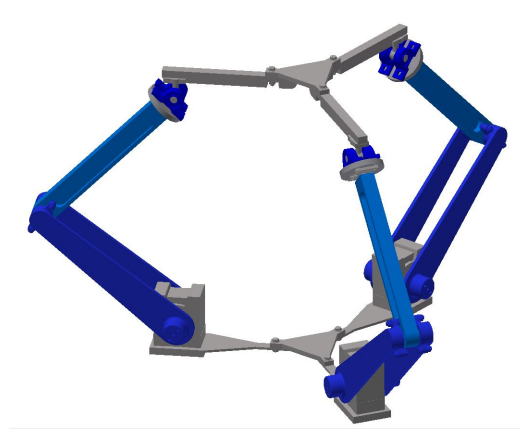

(a) CAD model

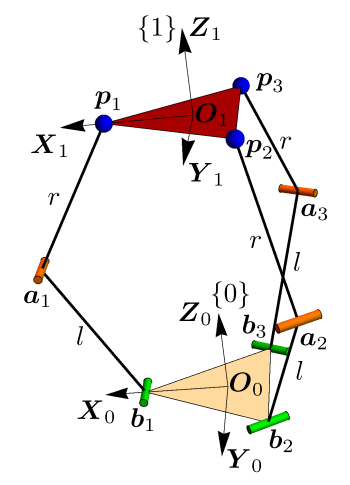

(b) Kinematic sketch

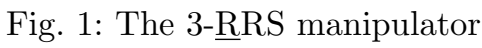

$$
\boldsymbol{b}_{1}=\{b, 0,0\}^{\top}, \quad \boldsymbol{b}_{2}=\boldsymbol{R}_{Z}(2 \pi / 3) \boldsymbol{b}_{1}, \quad \boldsymbol{b}_{3}=\boldsymbol{R}_{Z}(4 \pi / 3) \boldsymbol{b}_{1},
$$

where $\boldsymbol{R}_{Z}(\theta)$ indicates a CCW rotation through the angle $\theta$ about the positive $Z$ axis. The coordinates of the passive rotational joints are:

$$
\boldsymbol{a}_{i}=\boldsymbol{b}_{i}+\boldsymbol{R}_{Z}((i-1) 2 \pi / 3)\left\{l \cos \theta_{i}, 0, l \sin \theta_{i}\right\}^{\top}, i=1,2,3 .
$$

The vertices of the top platform, expressed in terms of the joint variables $(\boldsymbol{\theta}, \boldsymbol{\phi})$, are given by:

$$
\boldsymbol{p}_{i}=\boldsymbol{a}_{i}+\boldsymbol{R}_{Z}((i-1) 2 \pi / 3)\left\{r \cos \phi_{i}, 0, r \sin \phi_{i}\right\}^{\top}, i=1,2,3 .
$$

The vertices of the top platform can also be written in terms of the coordinates of the centre of the top platform $\boldsymbol{p}$, and the orientation of the top platform, given by the rotation matrix $\boldsymbol{R}_{p}$ with the Euler convention $\boldsymbol{R}_{Z}(\gamma) \boldsymbol{R}_{X}(\beta) \boldsymbol{R}_{Y}(\alpha)$ :

$$
\tilde{\boldsymbol{p}}_{i}=\boldsymbol{p}+\boldsymbol{R}_{p} \boldsymbol{R}_{Z}((i-1) 2 \pi / 3) \boldsymbol{a}, \text { where } \boldsymbol{a}=\{a, 0,0\}^{\top}, i=1,2,3 .
$$

Loop-closure equations for a parallel manipulator ${ }^{1}$ can always be written in the form:

$$
\boldsymbol{g}(\boldsymbol{\theta}, \boldsymbol{\phi}, \boldsymbol{x})=\mathbf{0}, \boldsymbol{g} \in \mathbb{R}^{m+n}, \boldsymbol{\theta}, \boldsymbol{x} \in \mathbb{R}^{n} \text {, and } \boldsymbol{\phi} \in \mathbb{R}^{m} .
$$

In the case of 3-RRS manipulator the Eq. (4) takes the form of three spatial vector loops:

\footnotetext{
${ }^{1}$ Over-constrained and redundant manipulators are not considered here.
} 


$$
\boldsymbol{g}_{i}=\tilde{\boldsymbol{p}}_{i}-\boldsymbol{p}_{i}, \quad i=1,2,3 .
$$

Eq. (5) relates the pose of the top platform to the joint variables. In the following section, these equations would be used to generate the different singularity conditions needed in the computation of the SWZ.

\section{Formulation and computation of the SWZ}

The SWZ of a parallel manipulator is bounded by the zero level-sets of four boundary functions, $S_{i}, i=1,2,3,4$ (see [4] for the details). The first two conditions, namely, the conditions for loss- and gain-type (refer to [4] and the references therein for the definitions of these singularities) singularities depend only on the kinematics of the manipulator, while the remaining two conditions, namely, link interference and joint limits, depend on the physical dimensions of the links as well. In this case, the conditions for joint limits are incorporated into the conditions for link interference, hence there are only three boundary functions to be computed for the identification of the SWZ. These functions are derived in the following.

\subsection{Loss-type singularity condition, $S_{1}=0$}

The condition for the loss-type singularities are derived first, as these demarcate the boundary of the workspace of the manipulator. In order to do that, first the passive joint variables, $\phi$, are eliminated from Eq. (4), leading to $\boldsymbol{h}(\boldsymbol{\theta}, \boldsymbol{x})=\left\{h_{1}, h_{2}, h_{3}\right\}^{\top}=\mathbf{0}, \boldsymbol{h}, \boldsymbol{\theta}, \boldsymbol{x} \in \mathbb{R}^{n}$, where:

$$
h_{i}=\left(\boldsymbol{p}_{i}-\boldsymbol{a}_{i}\right) \cdot\left(\boldsymbol{p}_{i}-\boldsymbol{a}_{i}\right)-r^{2}, \quad i=1,2,3 .
$$

Loss-type singularities occur where the branches of inverse kinematics merge. Applying the implicit function theorem (IFT), the condition for the same is obtained as $S_{1}=0$, where $S_{1}=\operatorname{det}\left(\frac{\partial \boldsymbol{h}}{\partial \boldsymbol{\theta}}\right)$. Fig. 2a shows such a singular pose of the manipulator.

\subsection{Gain-type singularity condition, $S_{2}=0$}

Gain-type singularities occur inside the workspace, and are characterized by the merger of the branches of forward kinematics. Therefore to derive the condition for the same, the platform pose variables $\boldsymbol{x}$ are eliminated from Eq. (5), to obtain an equivalent set of constraints involving only the active 


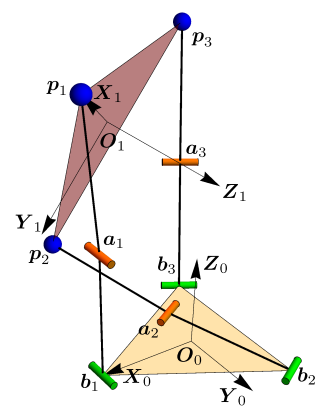

(a) Loss-type singularity

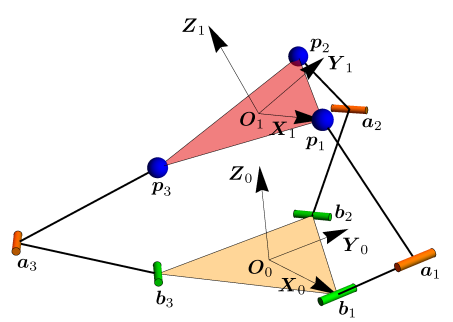

(b) Gain-type singularity

Fig. 2: Singular poses of the 3 - $\underline{R R S}$ manipulator (for the design parameters given in Section 4.)

and the passive joint variables:

$$
\begin{aligned}
& \boldsymbol{\eta}(\boldsymbol{\theta}, \boldsymbol{\phi})=\left\{\eta_{1}, \eta_{2}, \eta_{3}\right\}^{\top}=\mathbf{0}, \boldsymbol{\eta}, \boldsymbol{\phi} \in \mathbb{R}^{m}, \boldsymbol{\theta} \in \mathbb{R}^{n}, \text { where } \\
& \eta_{1}=\left(\boldsymbol{p}_{2}-\boldsymbol{p}_{1}\right) \cdot\left(\boldsymbol{p}_{2}-\boldsymbol{p}_{1}\right)-3 a^{2} \\
& \eta_{2}=\left(\boldsymbol{p}_{3}-\boldsymbol{p}_{2}\right) \cdot\left(\boldsymbol{p}_{3}-\boldsymbol{p}_{2}\right)-3 a^{2} \\
& \eta_{3}=\left(\boldsymbol{p}_{1}-\boldsymbol{p}_{3}\right) \cdot\left(\boldsymbol{p}_{1}-\boldsymbol{p}_{3}\right)-3 a^{2} .
\end{aligned}
$$

Invoking the IFT again, the condition for the gain-type singularity can be obtained as $S_{2}=0$, where $S_{2}=\operatorname{det}\left(\frac{\partial \eta}{\partial \phi}\right)$ Fig. $2 \mathrm{~b}$ shows one of the poses corresponding to the gain-type singularity.

\subsection{Link interference condition, $S_{3}=0$}

The task in this case is to identify the condition(s), such that the following can be stated: if $S_{3}(\boldsymbol{q})=0$, then the point $\boldsymbol{q}$ in the workspace of the manipulator can be attained in a manner such that the links do not interfere. Computation of $S_{3}$ is rather involved, as the physical dimensions of the links need to be taken into account. Certain simplification steps are included in the computation to reduce the CPU-time required. These are explained below. 


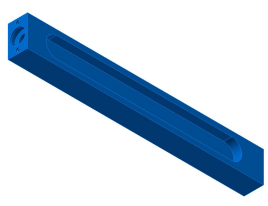

(a) Link-2 of a leg of the 3- RRS manipulator

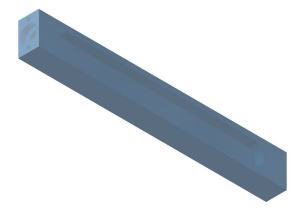

(b) Cuboid boundingbox

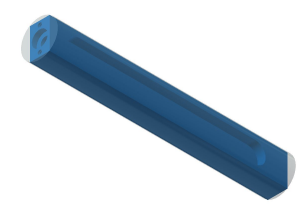

(c) Cylindrical

bounding-box

Fig. 3: Comparison of bounding-box shapes of the passive link of a leg of the of 3-RRS manipulator

\subsubsection{Geometric idealization of the links}

As seen in Fig. 1a, the links have complicated shapes. For example, the passive links have an I-shaped cross-section. Performing interference checks between two such links, while retaining all their geometric details, is typically done by tessellating the surface in terms of triangles, and then performing the interference checks between two sets of triangles. An approximation of the link geometry in terms of bounding boxes of simpler geometries can reduce the computational requirements drastically (see, e.g., [3]). Encapsulation of the links in such bounding boxes would, however, be essentially a conservative approximation, which would reduce the effective SWZ dimensions. Thus, it is essential to assess the packing efficiency, ${ }^{2}$ of the bounding boxes. Two different shapes, namely, the cuboid and the cylinder, are compared from this perspective, as shown in Fig. 3 for the case of the passive link in a leg. It is found that the packing efficiency is higher in the case of the cuboid, with a value of 0.541 , over the value 0.311 in the case of the cylinder. Hence, the cuboid is selected as the bounding box geometry in this work.

\subsubsection{Detection of collision between the bounding boxes}

In order to detect the interference between the cuboids, their faces are triangularized, as shown in Fig. 4. This reduces the task of detecting the interference of two cuboids to that of detection of intersections between two sets of twelve triangles each. This task is accomplished by using the Faster Triangle-Triangle Intersection Test ${ }^{3}$, which implements the algorithm presented in [1] Finally, it may be noted that not all cuboids need to be checked for interference, as some of them, e.g., $R_{11}$ and $R_{12}$ in Fig. 4 , need to remain in contact due to the joint between them, and therefore interference checks

2 Packing efficiency can be defined as the ratio of the volume of the link to the volume of its bounding box.

3 This code is available for free download at https://github.com/benardp/contours /tree/master/freestyle/view_map/triangle_triangle_intersection.c. 


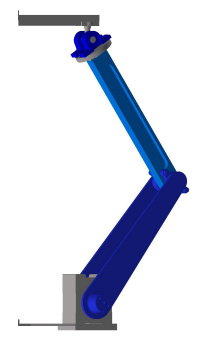

(a) Leg-1 of 3$\underline{R R S}$

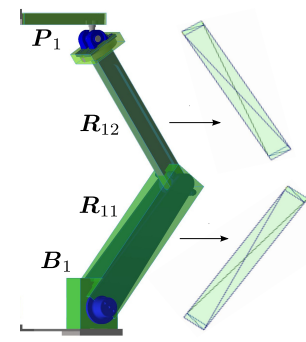

(b) Leg-1 of 3-RRS enclosed in cuboids

Fig. 4: Leg-1 of 3-RRS enclosed in cuboids

between these two are redundant. A collision list is developed to include in it all the cuboids that need to be checked. If all the required pairs are free of interference, then $S_{3}(\boldsymbol{q})=0$, and 1 otherwise.

These formulations are implemented in a $\mathrm{C}++$ code, and the results are presented in the following section.

\section{Numerical Results}

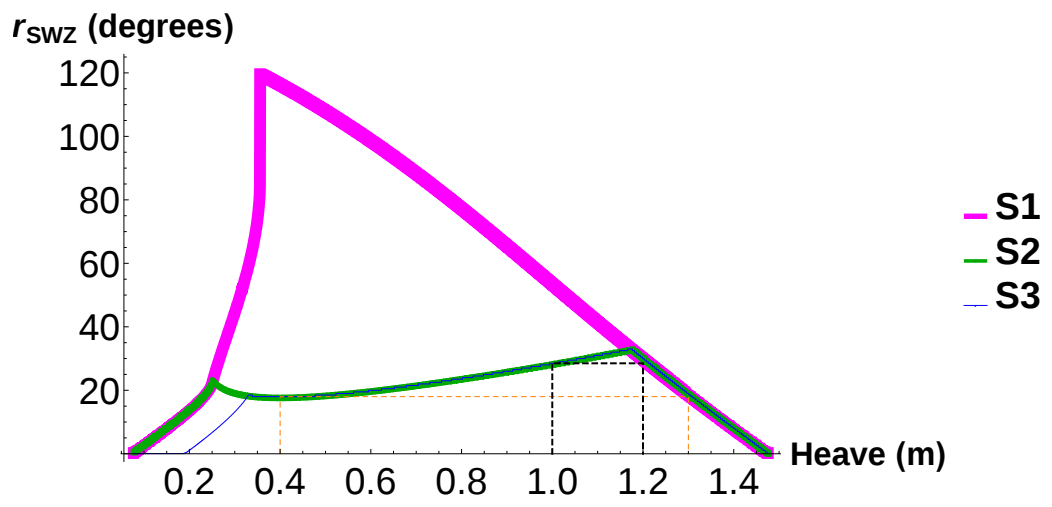

Fig. 5: Radius of SWZ circles at different values of $z(\mathrm{~m})$

The following are the dimensions of the 3 - $\underline{R} R S$ manipulator existing at the İzmir Institute of Technology ${ }^{4}: a=0.550, b=0.550, l=0.700, r=0.775$.

${ }^{4}$ All the linear dimensions are in metres and all angles are in radians, unless mentioned otherwise explicitly. 
Using these numerical values, the SWZ is computed in the following manner: 2-D slices of the workspace are considered parallel to the $\alpha-\beta$ plane, for $z \in$ $[0,1.475]$, at a resolution of $0.5 \mathrm{~mm}$. Each of these slices is scanned in either direction at a resolution of $0.125^{\circ}$, for the extent $\alpha, \beta \in\left[-180^{\circ}, 180^{\circ}\right]$. The SWZ, confined to this slice, is a circle of radius $r_{S W Z}$, centered at $\alpha=\beta=0$. The computation takes 41 minutes 58 seconds, while running on a single core of an Intel Core i7-4930K CPU with a processor speed of $3.40 \mathrm{GHz}$, and $64 \mathrm{GiB}$ of RAM. The results obtained are shown in Fig. 5. It can be seen how each of $S_{1}, S_{2}$ and $S_{3}$ bring $r_{S W Z}$ down progressively at each slice. The shaded area under the curve corresponding to $S_{3}$ represents the SWZ. However, as it is preferred to have a simple, convex representation of the SWZ, it is customary to fit a cylinder of desired shape inside this region. The height and radius of the cylinder can vary as per the choice of the application. Two such choices are shown in dotted lines in Fig. 5.

\section{Conclusion}

The paper presents the formulation and results pertaining to the computation of the SWZ of a spatial parallel manipulator, namely, an existing 3-RRS manipulator. The singularity conditions are derived from the loop-closure equations. Interference between the links are detected by using cuboidal bounding boxes, which are further discretized into triangles. The results obtained would be helpful in performing manipulation tasks safely inside the SWZ, without any further calculations, or need for validation of the robot's path for singularities or self-collision.

\section{References}

1. Devillers, O., Guigue, P.: Faster triangle-triangle intersection tests. Tech. Rep. RR-4488, INRIA (2002). URL https://hal.inria.fr/inria-00072100

2. Itul, T., Pisla, D.: 446. On the kinematics and dynamics of 3-dof parallel robots with triangle platform. Journal of Vibroengineering 11(1) (2009)

3. Perrin, N., Stasse, O., Lamiraux, F., Kim, Y.J., Manocha, D.: Real-time footstep planning for humanoid robots among $3 \mathrm{~d}$ obstacles using a hybrid bounding box. In: Robotics and Automation (ICRA), 2012 IEEE International Conference on, pp. 977-982. IEEE (2012)

4. Srivatsan, R.A., Bandyopadhyay, S.: Computational Kinematics: Proceedings of the 6th International Workshop on Computational Kinematics (CK2013), chap. Determination of the Safe Working Zone of a Parallel Manipulator, pp. 201-208. Springer Netherlands, Dordrecht (2014)

5. Tetik, H., Kalla, R., Kiper, G., Bandyopadhyay, S.: Position Kinematics of a 3-RRS Parallel Manipulator. Accepted for presentation at the 21st CISM-IFToMM Symposium on Robot Design, Dynamics, and Control, ROMANSY (2016) 